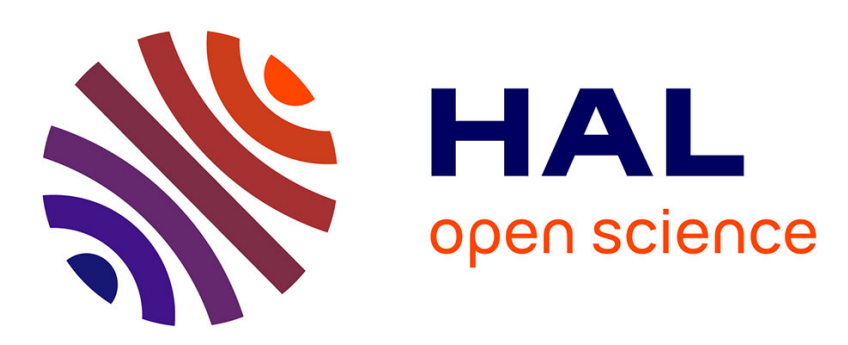

\title{
Quantification des populations de Sitona lineatus L. et de leurs attaques sur pois protéagineux (Pisum sativum L.) \\ Pierre Cantot, Fabrice Rolet
}

\section{To cite this version:}

Pierre Cantot, Fabrice Rolet. Quantification des populations de Sitona lineatus L. et de leurs attaques sur pois protéagineux (Pisum sativum L.). Agronomie, 1986, 6 (5), pp.481-486. hal-00884900

\section{HAL Id: hal-00884900 \\ https://hal.science/hal-00884900}

Submitted on 1 Jan 1986

HAL is a multi-disciplinary open access archive for the deposit and dissemination of scientific research documents, whether they are published or not. The documents may come from teaching and research institutions in France or abroad, or from public or private research centers.
L'archive ouverte pluridisciplinaire HAL, est destinée au dépôt et à la diffusion de documents scientifiques de niveau recherche, publiés ou non, émanant des établissements d'enseignement et de recherche français ou étrangers, des laboratoires publics ou privés. 


\section{Quantification des populations de Sitona linea- tus L. et de leurs attaques sur pois protéagineux (Pisum sativum L.)}

Pierre CANTOT

avec la collaboration de Fabrice RoLleT

I.N.R.A., Laboratoire de Zoologie, F 86600 Lusignan

RÉSUMÉ Le sitone du pois est très fréquent en France dans les cultures de pois protéagineux. Grâce à des enquêtes et
relevés effectués dans différents départements, on a défini une méthode simple de prélèvement des adultes par
piégeage à l'aide de boîtes posées sur le sol et des larves par prélèvement de terre. Une échelle de notation de 0
à 3 des dégâts occasionnés par les adultes hivernants sur les folioles de la première feuille des plantes de pois
est proposée. Cette notation est effectivement corrélée au nombre d'adultes présents dans le champ. La nota-
tion permet aussi de prévoir les effectifs larvaires ultérieurs. Les populations larvaires peuvent détruire
$90 \mathrm{p}$. 100 de la nodulation du pois lorsque leurs effectifs atteignent une douzaine de larves par plante.

Mots clés additionnels : Sitone, méthodes de prélèvements, notation d'attaque, relation capture-dégâts.

From field surveys, we investigated the relationship between the populations of wintering adults, their feeding on leaflets. the larval populations, and the number of newly emerged adults. The method to be used was the main subject of the research. Wintering adults were trapped using boxes put out every day in different parts of the field. The leaflet notches made by these adults were given a score from 0 to 3 . Soil samples were washed with a turbulent overflow process to evaluate larval populations per plant. The state of the nitrogen-fixing root nodules was noted. Emerging summer adults were trapped in the same type of box as the spring ones. The main quantitative relationship was the highly significant correlation between the spring score of adult notches on the leaflets of the first level of leaves and the subsequent larval populations. Other correlations were also analysed. Results are also given on damage caused by the larvae. In French pea crops, an adult score of 3 was related to a mean level of 12 larvae per plant and $90 \%$ of root nodules are destroyed.

Additional key words : Pea weevil, sampling methods, damage scoring, damage-capture relationship.

\section{INTRODUCTION}

Depuis 6 ans, les superficies consacrées à la culture du pois protéagineux de printemps n'ayant cessé de progresser (VAN HAECKE, 1984), il convenait de suivre de près l'évolution des populations d'insectes ravageurs présents dans cette culture et d'estimer leur importance pratique réelle. Des enquêtes réalisées grâce à la collaboration de différents organismes (I.N.R.A., F.N.A.M.S., S.P.V., I.T.C.F. $\left(^{*}\right)$ ) ont

$\left.{ }^{*}\right)$ F.N.A.M.S. : Fédération Nationale des Agriculteurs Multiplicateurs de Semences.

S.P.V. : Service de la Protection des Végétaux.

I.T.C.F. : Institut Technique des Céréales et Fourrages. permis de montrer que, parmi les ravageurs signalés dans les cultures de pois de printemps, notamment du bassin parisien, le sitone du pois (Sitona lineatus L.) occupe la première place (BOURNOVILLE, 1984).

La prise en compte par les agriculteurs et techniciens d'un ravageur tel que le sitone peut s'expliquer assez facilement. Les dégâts occasionnés par les adultes sur les jeunes cultures sont en effet très caractéristiques et assez bien visibles. Les éclosions estivales des insectes de la nouvelle génération, qui se produisent souvent non loin du moment de la récolte, sont parfois spectaculaires. Enfin, l'alimentation des larves aux dépens des nodosités fixatrices d'azote n'est pas non plus sans inquiéter les producteurs.

Ces constatations nous ont amenés à réaliser des 


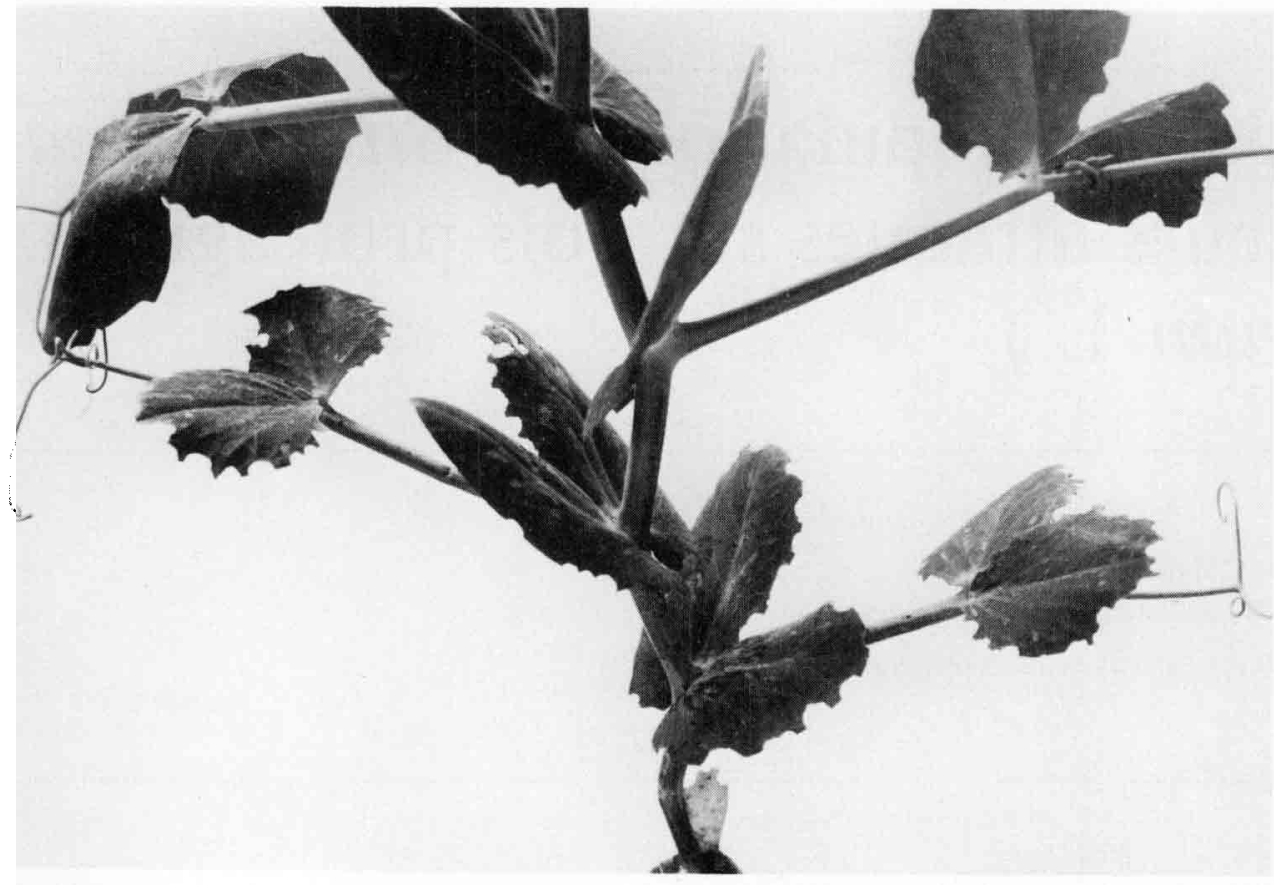

Figure 1

Dégâts d'adultes au printemps. Attack by S. lineatus adults.

(Photo S. Carré). observations précises afin de mieux situer l'importance des sitones dans les cultures françaises de pois protéagineux. Pour ce faire, il a fallu mettre au point différentes techniques de capture selon la période de l'année et le stade où se trouvent les insectes et évaluer les dégâts commis lors des différentes phases végétatives du pois. Nous les décrivons dans cette note avec, en préambule, une description rapide de la biologie de ces insectes afin de mieux situer les contraintes auxquelles nous nous sommes trouvés confrontés.

S. lineatus est un Coléoptère Curculionidae de 4 à $5 \mathrm{~mm}$ de long qui appartient à un genre étroitement inféodé aux légumineuses. Les adultes quittent les lieux d'hivernation en mars-avril et gagnent en volant les diverses légumineuses sur lesquelles ils s'alimentent ; mais, dès que le pois est levé, la culture est envahie de façon sélective. L'attaque des adultes sur les jeunes cultures est bien caractérisée par la présence d'encoches semi-circulaires sur le bord du limbe des folioles du pois (fig. 1). Ce type de dégâts est dû au comportement alimentaire de l'insecte qui se tient sur la tranche des feuilles en s'agrippant à leurs 2 faces. Après l'accouplement, les femelles déposent des œufs ovalaires de très petite taille $(0,3 \mathrm{~mm}$ de diamètre) essentiellement sur le sol, très peu sur les plantes. La fécondité des femelles est très élevée puisqu'on observe en élevage des pontes de plus de 1000 œufs (HoFFMANN, 1963). La durée d'incubation est de 12 à $13 \mathrm{j}$ à $20 \pm 1{ }^{\circ} \mathrm{C}$ (MARKKULA, 1959). Les jeunes larves sont très agiles et pénètrent rapidement dans le sol à la recherche des nodosités fixatrices d'azote dont elles s'alimentent obligatoirement puisqu'elles sont indispensables à leur survie. Après 5 stades larvaires (HAMON et al., 1984), la nymphose a lieu dans une petite coque terreuse située à quelques $\mathrm{cm}$ de la surface. Les nouveaux adultes émergent à partir de début juillet.

\section{MÉTHODES ET RÉSULTATS}

Nous examinerons successivement, les possibilités d'échantillonnage des adultes qui infestent la culture et leurs types de dégâts, les méthodes de prélèvement des larves et d'estimation de leur action sur les nodosités, les relations qui peuvent exister entre les populations de nouveaux adultes et les effectifs de larves ou d'adultes de printemps qui les ont précédées. Les données ont été recueillies à Lusignan (Vienne) pour les aspects méthodologiques et lors d'enquêtes dans différents départements, Aube, Côte-d'Or, Indre, Eure, Eure-et-Loir, Loiret, Vienne et Seine-et-Marne notamment.

\section{A. Les adultes hivernants}

Ils sont présents très tôt dans la culture où leur arrivée est échelonnée dans le temps. Il est très difficile, même pour des expérimentateurs avertis, d'observer ces adultes car ils sont mimétiques et se laissent tomber sur le sol à la moindre alerte. Par contre, les dégâts qu'ils commettent (encoches semi-circulaires) sont plus facilement décelables. Il convient donc de pouvoir compter les insectes présents dans le champ durant la phase de contamination et de noter d'une façon la plus fiable et la plus pratique possible les encoches réalisées sur les folioles par ces mêmes individus.

\section{Méthode de capture des adultes}

Parmi les diverses techniques utilisables, le filet fauchoir s'est avéré être peu propice pour leur capture. $\mathrm{Au}$ début du printemps, la plante est de petite taille et ne permet pas l'utilisation de cet instrument. En été, 
les adultes de la nouvelle génération restent le plus souvent localisés à la base de la végétation et ne sont pas capturés. L'aspirateur portable type «D. VAC » (DIETRICK, 1961) est en revanche utilisable ; il permet de quantifier les effectifs imaginaux à l'unité de surface. Dans le cadre des enquêtes, il s'avérait souhaitable de conseiller une technique simple qui permette la capture «en continu ". C'est pour cette raison que nous avons utilisé une boîte ouverte rectangulaire, en plastique (long. : $17 \mathrm{~cm}$, larg. : $11 \mathrm{~cm}$, haut. : $5 \mathrm{~cm}$ ) entièrement peinte en noir. Sur une des petites faces latérales, on perce un trou qui permet la communication avec une petite boîte ronde (diam. : $5 \mathrm{~cm}$ ) transparente dont le couvercle est grillagé. Dès que la culture de pois commence à lever, on pose 20 de ces boîtes à l'envers sur une ligne de semis prise au hasard au milieu du champ. On les enfonce très légèrement dans le sol afin qu'il n'y ait pas de communication avec l'extérieur. Les sitones adultes présents sur les plantules et dans les anfractuosités du sol se trouvent ainsi enfermés à l'obscurité ; ils se dirigent vers la lumière et entrent donc dans la petite boîte ronde où on les recueille facilement. Comme les arrivées d'adultes sur la culture sont progressives, il est nécessaire de changer les boîtes de place 1 à 2 fois par semaine afin de capturer les nouveaux arrivants. On utilise ces boîtes depuis la levée du pois jusqu'au stade $10-12 \mathrm{~cm}$ de haut, c'est-à-dire pendant une vingtaine de jours environ.

\section{Notation des dégâts sur plantules}

Chaque plante fait l'objet d'une notation d'après une échelle très simple d'estimation visuelle des dégâts (fig. 2) : 0 : pas d'attaque, $1:$ attaque faible, $2:$ attaque moyenne, 3 : attaque forte.

Pour tester la valeur de cette notation, nous avons utilisé un relevé effectué à une date où la gamme des
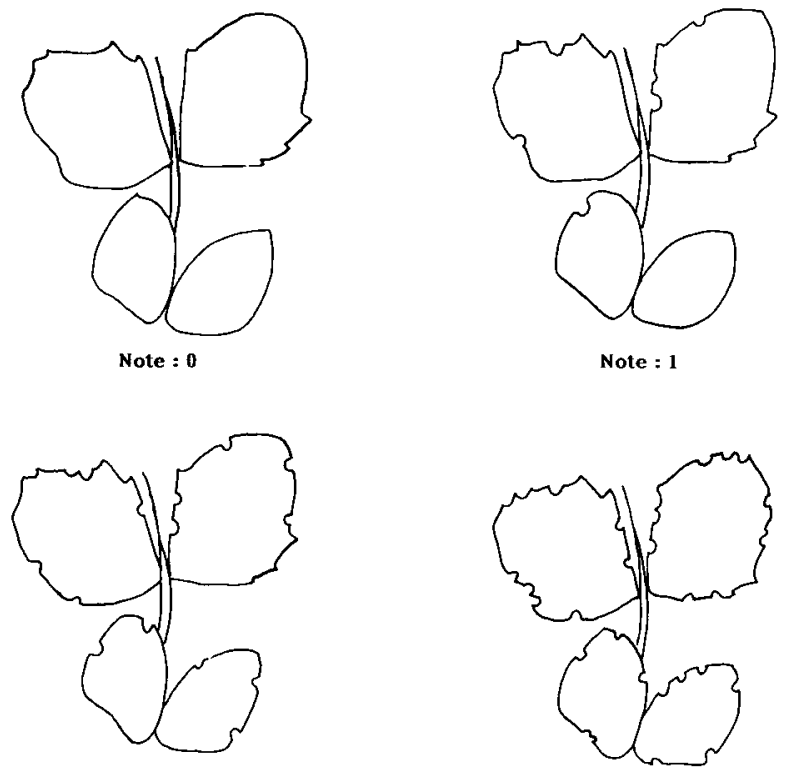

Note : 2

Note : 3

Figure 2

Echelle de notation visuelle utilisée pour la caractérisation des dégâts provoqués par les adultes de S. lineatus.

Scoring of leaf notches relative to the intensity of attacks by S. lineatus adults. différentes notes est assez large pour permettre une interprétation statistique. La notation visuelle est d'abord effectuée sur la $1^{\text {re }}$ feuille puis les encoches présentes sont dénombrées soit sur les stipules soit sur les folioles. Les notations ont porté sur chacune des plantes présentes dans les 20 boîtes de captures. En moyenne, nous avions 2,9 plantes par emplacement. Les résultats moyens obtenus sont mentionnès sur la figure 3 . La notation sur les folioles de la $1^{\text {re }}$ feuille vraie s'avère être en corrélation très hautement significative avec le nombre d'encoches effectivement présentes $(r=+0,87$ pour 19 d.d.1.). La relation est moins bonne ( $\mathrm{r}=+0,78$ pour 19 d.d.l.) lorsqu'on se base sur les stipules. Pour une plante notée 1 le nombre d'encoches sur l'ensemble des 2 folioles de la $1^{\text {re }}$ feuille est inférieur ou égal à 4 . Pour une notation 2, il faut au maximum 10 encoches et pour la note 3 un total de 11 encoches au moins doit être atteint. Les valeurs intermédiaires correspondent à la moyenne des notations qui ont été réalisées sur 20 plantes. Ces résultats permettent donc l'emploi de ce système de notation, plus simple qu'un comptage. Il sera utilisé lors des expérimentations ultérieures, notamment pour étudier la relation : captures d'adultes - notation.

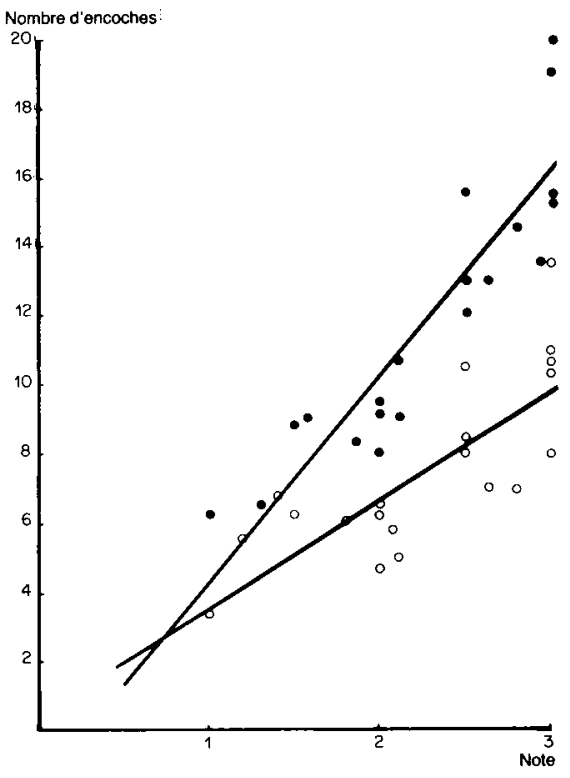

Figure 3

Relations entre la notation visuelle des attaques printanieres et le nombre d'encoches effectivement présentes sur les folioles (•) ou sur les stipules (O) de la $1^{\text {re }}$ feuille des plantes de pois. (I.N.R.A. Lusignan, pois « Finale 》, 1982).

Relationship between the scores for intensity of $\mathrm{S}$. lineatus adult attack and the number of notches of the first level of leaflets $(\bullet)$ and stipules (O).

\section{Relations : morsures alimentaires - effectifs d'adultes}

On les a déterminées de 2 façons :

\section{a) Evolution dans le temps}

On peut suivre l'évolution de la notation d'attaque et du nombre de sitones adultes en fonction de la date d'observation (fig. 4). La boîte de piégeage étant déplacée plusieurs fois durant la période de contamination de la culture, les insectes capturés à chaque 


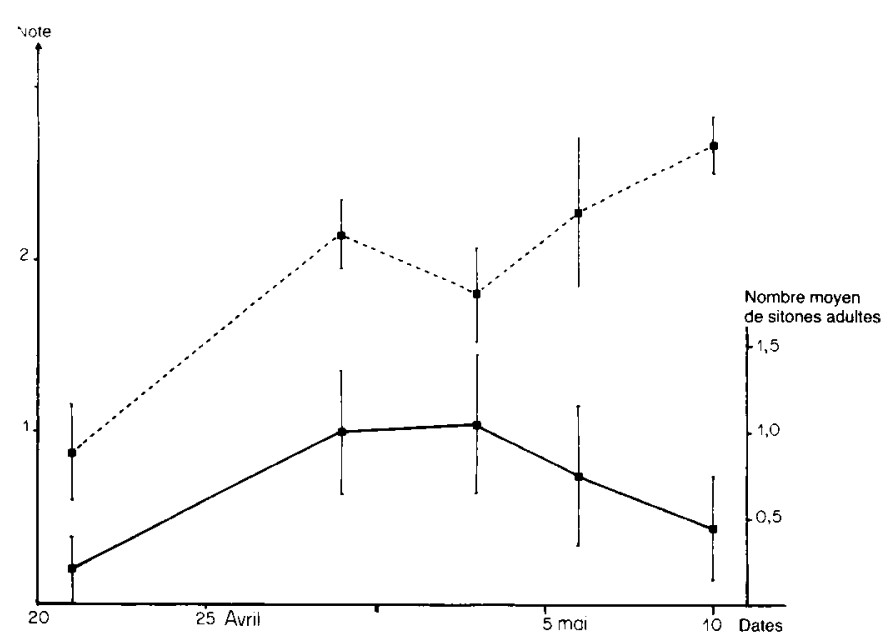

Figure 4

Evolution du nombre moyen de sitones adultes par boîte (trait plein) et de la notation visuelle des dégâts (trait pointillé) en plein champ. (I.N.R.A. Lusignan, pois « Finale », 1982).

Variation in the mean number of adults trapped (full line) and in the attack score (dotted line).

relevé correspondent à ceux déjà présents dans la culture, plus ceux, nouvellement arrivés entre les 2 dates d'observation. Quand les $1^{\text {ers }}$ adultes contaminants commencent à mourir, on assiste à une décroissance du nombre moyen d'adultes par boîte (du 5 au 10 mai). Par contre, à cette période, la note est maximum puisque les dégâts sur la $1^{\text {re }}$ feuille des 20 plantes prises au hasard dans le champ sont cumulés.

\section{b) Relation notation - effectifs d'adultes}

Grâce aux données d'enquêtes, les résultats recueillis dans 14 champs de 1982 à 1984 permettent d'établir la relation : notation moyenne maximum de morsures - effectif moyen maximum de sitones capturés à un moment situé durant le piégeage (fig. 5). Il existe une corrélation hautement significative $(\mathrm{r}=+0,89$ pour 13 d.d.1.). C'est-à-dire que plus la note maximum est élevée, plus l'effectif de sitones par boîte est important. Si l'on considère le nombre de sitones présents par plante et non par boîte, le coefficient de corrélation reste hautement significatif $(r=+0,79$ pour 10 d.d.l.). Cela nous permet d'établir qu'à une note supérieure à 2,5 correspond un effectif d'adultes supérieur à 0,5 par plante au moment du maximum de captures qui est l'ensemble des individus arrivés dans la culture depuis sa levée.

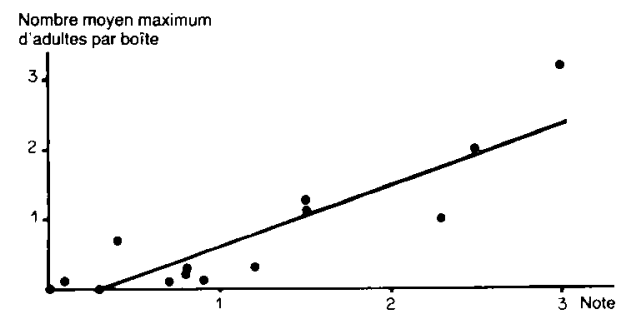

Figure 5

Relation entre la notation visuelle et le nombre moyen maximum d'adultes par boitte au printemps.

Relationship between the spring score and the number of adults trapped.
L'éventuel effet de serre provoqué par la boîte posée sur le sol n'est pas gênant ; au contraire, il permet une fuite rapide des adultes vers la boîte transparente aérée où ils sont recueillis facilement. Par contre, par temps frais, il arrive que quelques individus restent agrippés sur les parois internes de la boîte de piégeage.

\section{B. Les larves}

Les tout $1^{\text {ers }}$ stades larvaires se trouvent dans les nodosités qui sont vidées totalement de leur contenu ; plus tard, les larves étant plus grosses continuent à les ronger mais cette fois-ci de l'extérieur. Chez le pois, la majorité de ces nodosités se trouvent sur le pivot racinaire et les lésions occasionnées à la racine principale sont bien visibles (fig. 6).

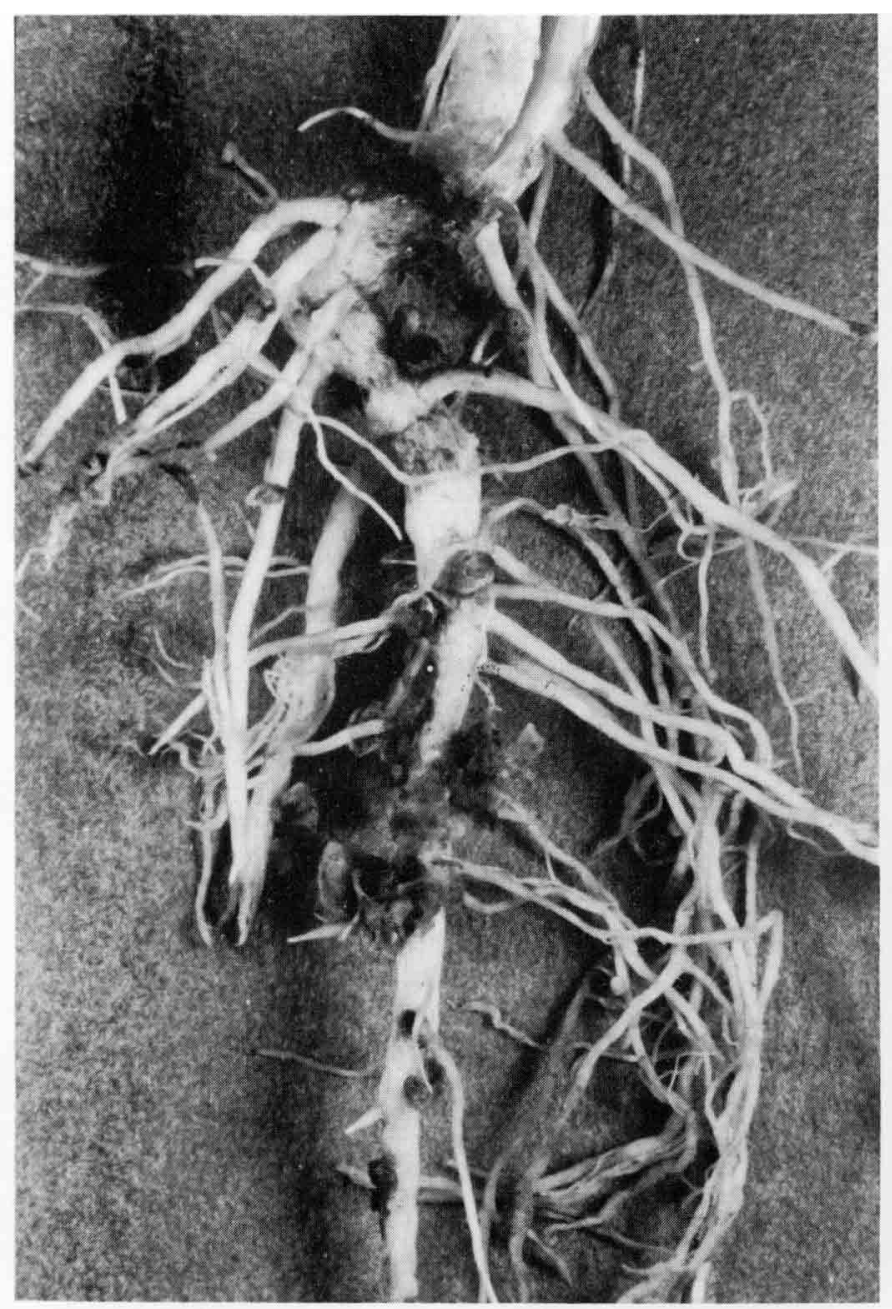

Figure 6

Dégâts larvaires sur les nodosités du pivot racinaire (Photo S. Carré).

Attack by S. lineatus larvae on the main root.

\section{Méthode d'échantillonnage des larves, nymphes et racines}

Les déprédations souterraines pouvant être importantes, il convient de rapprocher les effectifs de larves présentes dans le sol et l'intensité des dégâts. L'examen visuel d'une motte de terre contenant plus ou 
moins de racines étant difficile, il est nécessaire d'avoir recours au lavage de sol. La méthode employée est assez simple et un expérimentateur peut traiter une trentaine d'échantillons par jour. Les prélèvements au champ sont effectués à l'aide d'une pelle en $U$ sur des lignes de semis tirées au hasard. Chaque échantillon se compose d'une motte mesurant approximativement $20 \mathrm{~cm}$ de long, $10 \mathrm{~cm}$ de large et $15 \mathrm{~cm}$ de haut. Le maximum de larves se trouve dans les 15 premiers cm (MOULDEN, 1975). Les laveurs de terre que nous utilisons sont proches de ceux de SALT \& HolliCK (1944) et RAND (1973).

Pour une meilleure dispersion de l'échantillon dans l'eau, on agite légèrement celle-ci par un flux d'air arrivant en dessous du bac à décantation. L'air comprimé passe à travers un tamis à mailles très fines (diam. $0,20 \mathrm{~mm}$ ) afin d'être dispersé dans tout l'échantillon. Une arrivée tangentielle d'eau sous pression est située au niveau du filtre et permet d'avoir un bon brassage de l'échantillon.

Le déroulement du tri est le suivant : l'échantillon est prétraité dans une solution d'hexamétaphosphate de sodium à 2 p. 100 pendant plusieurs heures afin de disperser les colloïdes (BOUCHE \& BEUGNOT, 1972). Le tout est versé sur le crible où un jet d'eau permet une désagrégation complète et l'élimination des plus gros débris. C'est à ce moment que l'on met en marche le compresseur et l'arrivée d'eau dans le bac à décantation. Tous les éléments légers (végétaux et animaux), surnagent rapidement et lorsque le bac est plein, ils viennent se déverser dans le tamis de récolte. L'opération est arrêtée lorsque l'eau redevient limpide.

Les éléments récoltés sont ensuite éventuellement colorés au vert d'iode. La plupart des végétaux prennent donc une teinte foncée et les larves, restant blanches, sont facilement extraites même si la quantité de matières organiques est importante. Avec cette méthode, nous avons dénombré en moyenne, selon les années, de 30 à 40 nodosités par plante, surtout localisées sur le pivot racinaire.

\section{Evaluation des dégâts larvaires}

A chaque prélèvement sont estimés le nombre de nodosités dévorées ainsi que les effectifs larvaires. Les données acquises sont portées sur les figures 7 et 8 . Dans le $1^{\text {er }}$ cas, on constate que l'effectif larvaire maximum est de plus de 10 larves par racine en moyenne début juin et que les nodosités sont détruites à pratiquement 100 p. 100 . La légère diminution du nombre de larves par racine après la mi-juin est due à la nymphose et aux toutes $1^{\text {res }}$ sorties imaginables. La figure 8 représente les conséquences pour la nodulation de l'alimentation larvaire. Les racines et nodosités ont été examinées sous loupe binoculaire aux différentes dates et l'activité alimentaire des jeunes larves est très importante et rapide puisqu'on passe en $15 \mathrm{j}$. de 15 à 80 p. 100 de nodosités détériorées en moyenne par pied de pois.

\section{Les adultes de nouvelle génération}

Généralement vers la fin du mois de juin, lorsque les larves atteignent leur complet développement et se

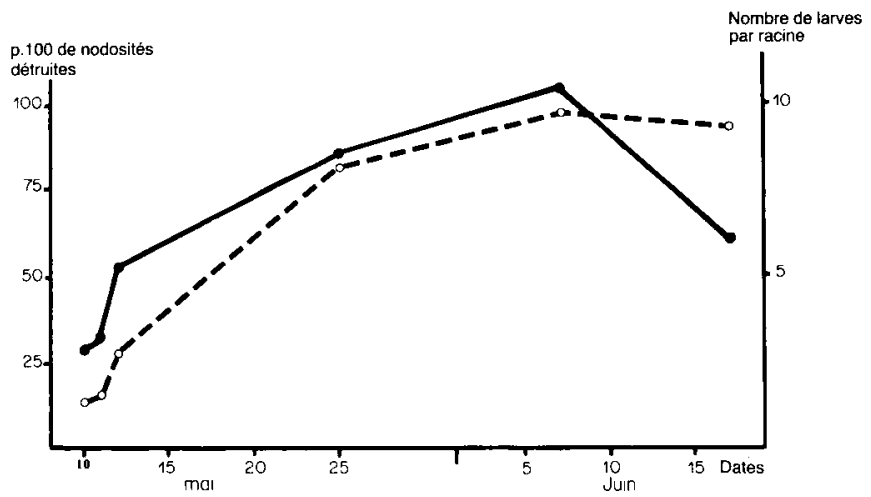

Figure 7

Evolution du nombre de larves de sitone du pois par racine (trait plein) et du pourcentage de nodosités détruites (trait pointillé) en fonction de la date de prélèvement. (I.N.R.A. Lusignan, pois "Finale », 1982).

Variation in the mean number of larvae per pea root (full line) and in the percentage of destroyed nodules (dotted line).

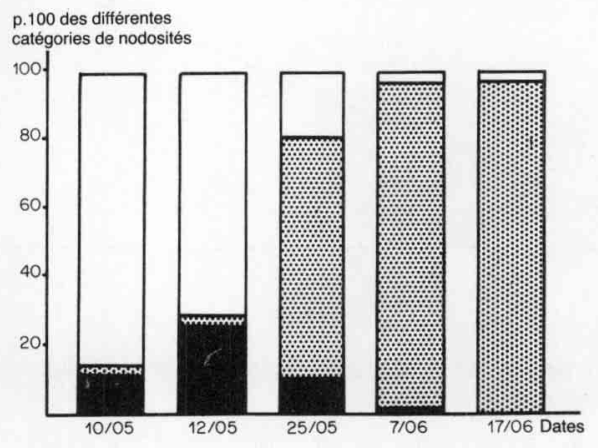

Figure 8

Etats des nodosités du pois en fonction de la date de prélèvement. (I.N.R.A. Lusignan, pois « Finale», 1982).

Condition of the nitrogen fixing root nodules.

Nodosités contenant des larves Nodules with larvae

$\because \because \because \because$ Nodosités détruites $\quad$ Destroyed nodules

Nodosités saines Sound nodules

nymphosent, on utilise de nouveau les boîtes-pièges décrites précédemment. On en place là aussi une vingtaine sur une ligne de semis, le pois ayant été préalablement coupé au ras du sol. Ces boîtes sont laissées en place jusqu'à la fin du mois de juillet. Les jeunes adultes sont recueillis quotidiennement dans la boîte transparente. Il existe une corrélation hautement significative ( $\mathrm{r}=+0,72$ pour 11 d.d.l.) entre le nombre moyen de larves par plante et l'effectif d'adultes de nouvelle génération recueillis par boîte de piégeage.

\section{DISCUSSION ET CONCLUSION}

Les différentes techniques rapportées ci-dessus permettent d'apprécier les populations de $S$. lineatus présentes à un moment donné dans les cultures de pois protéagineux, que ces insectes soient au stade adulte ou larvaire. Il est surtout intéressant de constater qu'il existe une suite de corrélations positives hautement 
significatives, c'est-à-dire que la notation de printemps permet de déduire le nombre d'adultes hivernants présents par plante mais aussi, comme le montre la figure 9, le nombre de larves par plante qui pourront se développer plus tard $(r=+0,80$ ths pour 11 d.d.l.). La relation linéaire est encore significative $(r=+0,53$ pour 13 d.d.l.) entre le nombre d'adultes de nouvelle génération à l'unité de surface et la note printanière. Cette moins bonne relation s'explique par le fait qu'il est nécessaire de bien situer l'époque d'installation des boîtes afin de couvrir l'ensemble de la période d'apparition des nouveaux adultes. Ces conditions ont été plus difficiles à remplir dans le cadre de nos enquêtes.

Les travaux menés en Grande-Bretagne par BARDNER et al. (1979), Mc EWEN et al. (1981), KING (1981)

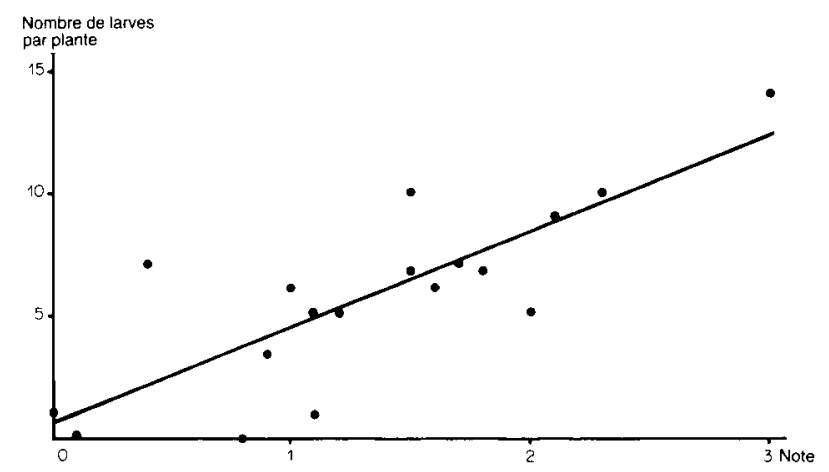

Figure 9

Relation entre la note d'attaque printanière des adultes et le nombre de larves par plante en été.

Relationship between the spring scoring and the number of larvae per plant in summer. n'arrivent pas aux mêmes conclusions que les nôtres. Le système de notation des morsures établi par ces auteurs n'est pas effectué à un niveau constant de la plante. Il prend en effet en considération les 2 dernières feuilles développées au moment de la notation. D'autre part, les conditions climatiques différentes peuvent peut-être interférer sur l'intensité d'alimentation des adultes contaminants et sur la coïncidence entre la présence de l'insecte et la phénologie de la plante. Dans notre cas, l'application d'une méthode extrêmement simple (notation des morsures de l'attaque printanière) permet de prévoir les populations larvaires ultérieures. Leurs effectifs souvent élevés peuvent détruire totalement la nodulation du pois.

Dans une nouvelle étape, nous nous proposons de quantifier les conséquences de cette destruction sur les facteurs de rendement du pois protéagineux. Les 2 possibilités pour la plante de subvenir à ses besoins azotés (fixation symbiotique ou prélèvement direct dans le sol) imposent de se prémunir contre les interférences sol-plante. Il n'empêche que même si la plante n'a pas à souffrir directement du manque de fixation, l'alimentation larvaire des sitones pose à nouveau le problème de l'économie de fertilisants azotés après une culture de légumineuses.

Reçu le ler juillet 1985. Accepté le 27 janvier 1986.

\section{REMERCIEMENTS}

Nous tenons à remercier tout particulièrement les différentes personnes de la F.N.A.M.S., de l'I.T.C.F. et du S.P.V. qui ont participé à l'exécution des différents protocoles. Nos remerciements vont aussi à R. BourNoviLle (I.N.R.A., Lusignan) pour les corrections apportées au manuscrit.

\section{RÉFÉRENCES BIBLIOGRAPHIQUES}

Bardner R., Fletcher K. E., Griffiths D. C., 1979. Problems in the control of the pea and bean weevil (Sitona lineatus). Proc. $1979 \mathrm{Br}$. Crop Prot. Conf., Pests and Diseases, 223-229.

Bouché M. B., Beugnot M., 1972. Contribution à l'approche méthodologique de l'étude des biocénoses. II. L'extraction des macroéléments du sol par lavage tamisage. Ann. Zool. Ecol. anim., $4,537-544$.

Bournoville R., 1984. Insectes nuisibles aux protéagineux, situation actuelle, organisation de la lutte, 199-217. Journ. int. Protéagineux, Paris 12 déc. 1984, 311 p.

Dietrick E. J., 1961. An improved back pack motor pan for section sampling of insect populations. J. econ. Entomol., 54, 394-395.

Hamon N., Allen-Williams L., Lee J. B., Bardner R., 1984. Larval instar determination of the pea and bean weevil Sitona lineatus L. (Coleoptera : Curculionidae). Entomol. Mont. Mag., 120, 167-171.

Hoffmann A., 1963. Les Sitona, 929-940. In Balachowsky A. S. : "Entomologie appliquée à l'agriculture ". Masson Ed., 1, 1391 p.

King J. M., 1981. Experiments for the control of pea and bean weevil (Sitona lineatus) in peas, using granular and liquid insecticides. Br. Crop Prot. Counc., 327-331.
McEwen, J., Bardner R., Briggs G. G., Bromilow R. H., Cockbain A. J., Day J. M., Fletcher K. E., Legg B. J., Roughly R. J., Salt G. A., Simpson H. R., Webb R. H., Witty J. F., Yeoman D. P., 1981. The effects of irrigation, nitrogen, fertilizer and the control of pests and pathogens on spring-sown field beans (Vicia faba $\mathbf{L}$.) and residual effects on two following winter wheat crops. J. Agric. Sci. Camb., 96, 124-150.

Markkula M., 1959. The biology and especially the oviposition of the Sitona Germ. (Col. Curculionidae) species occuring as pests of grassland legumes in Finland. Publ. Fin. State Agric. Res. Board, 178, 41-74.

Moulden J., 1975. Sampling methods and life table date for Sitona humeralis Steph. Dep. Agric. S. Aust. Agric. Branch Rep., 62, $1-48$.

Rand J. R., 1973. A turbulent overflow process for extracting weevil larvae (Coleoptera : Curculionidae) from large soil samples. $J$. aust. entomol. Soc., 12 (3), 225-229.

Salt G., Hollick F. S. J., 1944. Studies of wireworm populations. 1. A census of wireworms in pasture. Ann. appl. Biol., 31, 53-64.

Van Haecke Y., 1984. Pois, féverole, lupin : une voie vers la réduction de notre déficit en matière protéiques, 3-8. Journ. int. Protéagineux, Paris 12 déc. 1984, 311 p. 